\title{
Sexually Transmitted Diseases Among Residents in Nursing Homes: The Nurses' Perspective
}

\author{
Lauren N. Maziarz'; Nikki Sorgi ; Nicole Fischer ${ }^{1}$ \\ ${ }^{1}$ Department of Public and Allied Health, Bowling Green State University, Bowling Green, $\mathrm{OH}$ \\ Corresponding Author: Lauren Maziarz, Department of Public and Allied Health, Bowling Green State University, Bowling Green, OH 43403, \\ Imaziar@bgsu.edu
}

Submitted November 9, 2020 Accepted April 8, 2021 Published June 21, 2021 https://doi.org/10.18061/ojph.v4i1.8069

\begin{abstract}
Background: Examining registered nurses' perceptions of sexually transmitted disease (STD) education among residents in nursing homes allows for broader insight into why STDs continue to increase among older adults.

Methods: A 4-page pilot survey was mailed to nursing home directors of nursing in Northwest Ohio $(n=99)$ with a response rate of $32 \%$. Directors of nursing were the target population as they are the most likely employee to hold registered nurse licensure. The health belief model formed the basis for the survey.
\end{abstract}

Results: Most nurses did not see STDs or human immunodeficiency virus (HIV) as problems among their residents (100\% and 96\%, respectively) yet support for sex among residents was high. All (100\%) agreed sex among married residents should be supported, while $77 \%$ agreed sex among nonmarried residents should be supported. Most nurses stated they were comfortable discussing HIV risk (84\%), STD risk (84\%), erectile dysfunction (75\%), sexual desire and intimacy (72\%), and correct condom use (66\%). The most commonly reported perceived barriers to STD education were family opposition (63\%), resident embarrassment (56\%), and lack of education regarding the prevalence of STDs in older adults (53\%). The most commonly reported perceived benefits to STD education were being seen as a leading facility for healthy initiatives (66\%) and promotion of healthy sexual relationships among residents (56\%).

Conclusion: There was strong support for STD education among nurses though implementation remains rare. Addressing the most commonly perceived barriers and benefits may prove beneficial in increasing the number of LTCFs that provide STD education to residents.

Keywords: Sexually transmitted disease education; STDs; Nursing homes; Nurses' perceptions

\section{INTRODUCTION}

Many older adults in the United States (US) aged 65 to 80 years are sexually active $(40 \%)^{1}$ yet report less condom use, fewer sexually transmitted disease (STD) screenings, and lower risk perceptions of STDs than their younger counterparts. ${ }^{2}$ While STD burden in the US remains highest during adolescence, STDs are on the rise among older adults. ${ }^{3}$ Rates for herpes, gonorrhea, syphilis, chlamydia, hepatitis B, and trichomoniasis rose 23\% between 2014 and 2017 for those over age 60.3 Unfortunately, discussions with care providers about sexual health remain rare. The majority of older adults (62\%) say they would talk to their health care provid- er if they were concerned about their sexual health, yet only $17 \%$ have done so in the last 2 years. ${ }^{1}$

Nurses who work in long-term care facilities (LTCFs) represent a unique population for studying barriers to STD education among older adults. Elderly individuals who reside in LTCFs tend to have higher dependency on care staff than the rest of the population, potentially hindering opportunities for sex and sexual expression. 4,5 Moreover, an increased prevalence of chronic illnesses compared to the general population coupled with prescription medications that limit sexual drive also contribute to limited opportunities for sexual intimacy.4 This does not mean that individu- 
als in LTCFs are asexual, however, as research has shown that sexual intimacy, while possibly becoming more difficult in older age, is still an important aspect of life.5,6 Since more than 1.5 million nurse employees (ie, registered nurses [RNs], licensed practical nurses [LPNs], and licensed vocational nurses [LVNs]) work in LTCFs in the US,7 examining barriers to STD education from the nurses' perspective allows for insight into why STD education and screening in nursing homes remains rare among this vulnerable population.

Although sexual activity decreases with age, interest in sex remains strong with $65 \%$ of adults aged 65 to 80 years reporting that sex is important to their overall quality of life. ${ }^{1}$ While these findings do not specify whether the adults are living in LTCFs, older men and women who self-report being in fair or poor health still acknowledge having regular sex at least 2 to 3 times per month (46\% versus $56 \%$, respectively). ${ }^{8}$ Several studies have also documented the assumption among LTCF staff that sexual expression over a certain age is inappropriate, ${ }^{4,9}$ which is alarming given that sexual activity does not decrease in older adulthood to the extent to which is commonly perceived. Nurses and other health care personnel are typically seen as being responsible for sex education for the elderly, although training is routinely focused on the physical aspects of sex as opposed to the psychological or emotional issues of intimacy. ${ }^{10}$ Staff regularly report either seeing sexual activity in LTCFs as distracting or even disturbing to younger family members and staff.4,9 Care providers may also feel as though sexual education is low on a long list of priorities, as comorbidities are common and dependence on staff is high. ${ }^{9}$

Negative staff attitudes regarding sexual expression also contribute to a lack of open dialogue among residents, as older adults are less likely to talk about sex if their health care providers do not broach the subject first. ${ }^{8}$ Active listening during conversations and connecting with patients to better understand the appropriate times when sexual health may be explained openly are important skills all health care professionals should acquire.4,9,11,12 It can be argued that the nature of longer and more meaningful encounters between a medical provider and their elderly resident may prove more effective for discussing sexual health than briefly visiting with shallow conversations, ${ }^{13}$ making LTCFs a place to engage in meaningful dialogue with residents regarding their sexual expression.

Previous research has demonstrated that there is a significant gap in knowledge and self-efficacy among care providers when it comes to addressing STDs in the elderly.4,14-19 One well-shared viewpoint, however, expresses the notion that more education on sexual health may yield more on-the-job confidence thereby reducing hesitation when approaching a person of any age concerning the topic.4,9,10,12,17-19 Knowing and understanding the sexual health needs of older adults makes care providers more likely to introduce the topic of sex into their everyday conversations. In a recent Australian study, nurse training has been found to be effec- tive at improving LTCF nurse knowledge and attitudes regarding older adult's sexuality, ${ }^{20}$ pointing to the need to expand knowledge and training for US-based LTCF nurses.

Facility policies can also either hinder or enhance communication regarding sexual health. ${ }^{12}$ Just $23 \%$ of LTCFs in the US have policies related to sexual expression, and even fewer (13\%) provide staff training on intimacy and sexual behavior. ${ }^{19}$ Without policies to guide the residents, there are no strict rules to govern the health care staff and to shape their perspectives when it comes to sexual expression. ${ }^{12}$ Several studies have also discussed how the lack of privacy among residents, family member involvement in decision making, as well as a lack of a partnercan all hinder sexuality among residents. ${ }^{4,5}$ Unfortunately, there is no published data on the percentage of LTCFs that provide STD education or screening, making it difficult to ascertain how common the practice is.

Nurses and care providers in LTCFs play an integral role in shaping the day-to-day lives of residents. Since resident dependency is high and autonomy is limited, this study represents an important step to understanding how nursing homes may differ from other populations in terms of STD prevention campaigns. As one of the first systematic quantitative explorations of RNs to examine perceptions of STD education in LTCFs, gaining insight into the barriers to and benefits of STD education will inform targeted education campaigns and aid in the development of evidence-based training programs for nurses in the future.

\section{METHODS}

\section{Setting and Design}

This study used a cross-sectional study design to explore nurses' beliefs and attitudes towards sexual relationships and STDs in nursing home residents. The health belief model ${ }^{21,22}$ formed the theoretical basis to explore nurses' perceived barriers, perceived benefits, and beliefs regarding both sexual intimacy and STDs among residents. The Health Belief Model was chosen to guide this study as other research has demonstrated the theory's applicability to examining perceptions, barriers, and benefits at the systems level.23,24 The 21-item instrument included 13 close-ended questions, 7 demographic items, and 1 open-ended question for additional insights. To ensure content validity, literature in the areas of sexual health, STD prevention, and health care provider knowledge provided the basis for question development. $2,4-6,9,12,17,18,20$

\section{Participants}

The subjects used for this study consisted of directors of nursing working in nursing homes in Northwest Ohio. Directors of nursing were the target population as they are the most likely employee to hold RN licensure, and, in fact, may be one of the only RNs in the facility. Thus, targeting directors of nursing was the most effective way of gathering RN perceptions. Furthermore, directors of nursing can help shape and direct policies and procedures within LTCFs, meaning their opinions can either support or hinder 
change within an agency. ${ }^{12}$ All 99 nursing homes in the 10 counties within Northwest Ohio received surveys. Facility names and locations were compiled from the Medicare Nursing Home Compare website and cross-referenced with the local Area Office on Aging (AOA) database. It should be noted that the term LTCF can encompass all long-term care options, including independent living facilities with minimal to no nurse support but can also encompass nursing homes with complete resident dependence. Since assisted living facilities are not well-defined by the State of Ohio, agencies listed only as an assisted living facility according to the AOA were excluded from this study.

\section{Procedures}

This research study was approved by the primary investigator's institutional review board. Mailed surveys were sent over 3 waves, all 2 weeks apart. Starting in June 2019, the initial wave included a hand-signed cover letter with an explanation of the research, a \$1 bill as an incentive, and a stamped return envelope sent through first-class mail. The second wave included a postcard requesting participants who had not already done so to complete the survey.

The third and final wave included another copy of the survey with a cover letter sent to the participants who had not yet completed the form.

\section{Data Analysis}

Data were analyzed using IBM SPSS Statistics 24.0 for Mac. We used descriptive analyses to examine the percentage of participants who responded to given survey items. Chi-square analyses were used to compare responders and nonresponders on several variables to determine sample representativeness.

\section{RESULTS}

\section{Demographics}

Out of 99 mailed surveys, a total of 32 surveys were returned for a response rate of $32 \%$. The majority of respondents identified as female (94\%), aged 40 and over (76\%), White (81\%), with an associate's degree as the highest level of education (53\%), 1 to 5 years of experience at their current facility (47\%), and came from midsized facilities with 50 to 99 beds (47\%). Most respondents also identified their current job title as the director of nursing (88\%) (Table 1). The 2 respondents who did not identify as a

\section{Table 1. Demographic and Background Characteristics of Respondents}

\begin{tabular}{|c|c|}
\hline Item & $\mathbf{N}(\%)$ \\
\hline \multicolumn{2}{|c|}{ Number of beds in the facility } \\
\hline$<50$ & $5(16)$ \\
\hline $50-99$ & $15(47)$ \\
\hline 100-199 & $11(34)$ \\
\hline \multicolumn{2}{|l|}{ Gender } \\
\hline Female & $30(94)$ \\
\hline Male & $1(3)$ \\
\hline \multicolumn{2}{|l|}{ Age } \\
\hline $20-29$ & $2(6)$ \\
\hline $30-39$ & $5(16)$ \\
\hline $40-49$ & $12(38)$ \\
\hline $50+$ & $12(38)$ \\
\hline \multicolumn{2}{|l|}{ Race/ethnicity } \\
\hline White & $26(81)$ \\
\hline Non-white & $5(16)$ \\
\hline \multicolumn{2}{|c|}{ Highest level of education } \\
\hline Diploma program & $1(3)$ \\
\hline Associate's degree & $16(53)$ \\
\hline Bachelor's degree & $13(41)$ \\
\hline Master's degree & $1(3)$ \\
\hline \multicolumn{2}{|l|}{ Years at current facility } \\
\hline $1-5$ & $15(47)$ \\
\hline $6-10$ & $6(19)$ \\
\hline $11-15$ & $2(6)$ \\
\hline $16-20$ & $1(3)$ \\
\hline $20+$ & $7(22)$ \\
\hline \multicolumn{2}{|l|}{ Current job title } \\
\hline Director of nursing & $28(88)$ \\
\hline Other & $2(6)$ \\
\hline
\end{tabular}

Note: Percentages may not equal $100 \%$ due to rounding and/or nonreported answers. 
director of nursing identified their positions as administrative in nature ("regional resource nurse manager" and "administrator") and were included in the results.

Additionally, to assess sample representativeness, responders and nonresponders were compared based on several variables reported in the skilled nursing facility provider catalog which includes publicly available data reported by nursing homes. ${ }^{25}$ Pearson chisquare results indicate no significant difference between responders and nonresponders depending on which county the facility was located in (Pearson $\left.\chi^{2}(9, N=99)=15.77, p=.072\right)$, the number of beds in the facility (Pearson $\chi^{2}(3, \mathrm{~N}=90)=1.50, \mathrm{p}=.682$ ), and the Centers for Medicare and Medicaid Services (CMS) RN staffing rating, which rates the number of adjusted RN hours per resident per day (Pearson $\left.\chi^{2}(4, \mathrm{~N}=76)=1.39, \mathrm{p}=.845\right)$. Table 2 provides a comparison of these variables among both responders and nonresponders as well as all nursing homes in Ohio.

\section{Sex Education Practices, Condom Availability, and Training}

The vast majority of respondents (94\%) stated there is no sex education provided to residents at their facility, while only $13 \%$ stated condoms are provided to residents who are sexually active. When asked if they had been trained on how to address STDs among residents, $87 \%$ said they had no formal training. Among those that had been trained $(n=4)$, education had occurred through either their nursing education $(n=2)$, employee education $(n=1)$, or continuing education $(n=1)$.

Interestingly, when asked whether STDs were a significant health problem among residents in their facility, all (100\%) respondents disagreed with this statement. In contrast, when asked if STDs were a significant problem among older adults in the US, only 53\% disagreed with this statement. Disparities also existed in perceptions of HIV burden. Most (97\%) disagreed HIV was a significant problem among residents at their facility, while only $38 \%$ disagreed HIV was a significant problem among older adults in the US.

\section{Facilitation of Sex and Sexual Relationships}

Respondents were unanimously supportive (100\%) of sex among married residents at their facility, while slightly fewer (77\%) agreed sex among nonmarried residents should be supported. The majority (78\%) also agreed sex among LGBTQ+ residents should be supported. Moreover, most (87\%) believed their facilities should provide private spaces for intimate partner visits, but fewer (52\%) believed condoms or lubricants (52\%) should be provided for residents who are having sex.

\section{Table 2. Comparison of Responders to Nonresponders/Ohio Nursing Homes}

\begin{tabular}{|c|c|c|c|}
\hline Characteristic & Responders N (\%) & Nonresponders N (\%) & Ohio nursing homes $\mathbf{N}(\%)^{a}$ \\
\hline \multicolumn{4}{|c|}{ Number of beds in facility } \\
\hline$<50$ & $5(16)$ & $18(20)$ & $157(17)$ \\
\hline $50-99$ & $15(47)$ & $51(57)$ & $504(53)$ \\
\hline $100-199$ & $11(34)$ & $20(22)$ & $280(29)$ \\
\hline$>199$ & $0(0)$ & $1(1)$ & $11(1)$ \\
\hline \multicolumn{4}{|c|}{ Registered nurse (RN) staffing rating ${ }^{b}$} \\
\hline 1 star & $2(8)$ & $3(6)$ & $77(9)$ \\
\hline 2 stars & $9(35)$ & $19(37)$ & $289(35)$ \\
\hline 3 stars & $10(39)$ & $19(37)$ & $268(32)$ \\
\hline 4 stars & $4(15)$ & $5(10)$ & $139(17)$ \\
\hline 5 stars & $1(4)$ & $5(10)$ & $64(8)$ \\
\hline \multicolumn{4}{|c|}{ County of facility } \\
\hline Lucas & $9(28)$ & $35(52)$ & \\
\hline Erie & $5(16)$ & $3(5)$ & \\
\hline Wood & $5(16)$ & $8(12)$ & \\
\hline Sandusky & $4(13)$ & 7 (10) & \\
\hline Ottawa & $3(9)$ & $1(2)$ & \\
\hline Henry & $3(9)$ & $1(2)$ & \\
\hline Fulton & $1(3)$ & $4(6)$ & \\
\hline Paulding & 1 (3) & $1(2)$ & \\
\hline Williams & $1(3)$ & $3(5)$ & \\
\hline Defiance & $0(0)$ & $4(6)$ & \\
\hline
\end{tabular}

Note: Percentages may not equal $100 \%$ due to rounding and/or nonreported answers. ${ }^{\mathrm{a}} \mathrm{N}=953$

${ }^{\mathrm{b}} \mathrm{RN}$ staffing rating is based on a 1-5 point scale that rates the number of adjusted RN hours per resident per day. The lower the rating, the fewer RN hours per resident per day. 
As a whole, nurses were supportive of talking with residents about their sexual health. The majority of nurses agreed they should provide education to residents on the risks of engaging in sex $(85 \%)$, talk with their residents about their sexual health needs (84\%), and support sexual intimacy among residents (75\%). When asked about sexual desire among residents, $72 \%$ of nurses agreed sexuality was a lifelong need among residents, and only $44 \%$ of nurses agreed residents in their facility desired sexual intimacy. Nurses did not perceive residents as being open to discussions on sex, however, with only $9 \%$ agreeing residents would want to talk about sexual intimacy with staff.

\section{Perceived Barriers and Benefits to Providing STD Education}

The most commonly reported perceived barriers to STD education were family opposition (63\%), resident embarrassment (56\%), lack of education regarding the prevalence of STDs in older adults (53\%), and not having enough time to provide education to residents $(50 \%)$ (Table 3 ). The most commonly reported perceived benefits to STD education were being seen as a leading facility for healthy initiatives $(66 \%)$, promotion of healthy sexual relationships among residents (56\%), improved overall health among residents $(47 \%)$, and improved resident knowledge of STDs (47\%) (Table 4).

\section{Perceived Support and Opposition to STD Education}

The vast majority of nurses agreed they are personally supportive of STD education for residents (97\%), and 75\% also believed residents would be supportive. In terms of other health care professionals, most believed LTCF administrators (78\%), physicians (78\%), and nurses (56\%) would all be supportive individuals, while fewer (50\%) agreed nursing assistants would be supportive. Of the responses listed, family members of residents were the only group noted as being opposed to STD education by the majority of respondents $(66 \%)$.

\section{Comfort with Sexual Health Discussions}

The respondents were asked how comfortable they would be discussing certain sexual health topics with residents. Most stated they were comfortable discussing STD risk (84\%), HIV/AIDs risk (84\%), erectile dysfunction (75\%), sexual desire and intimacy (72\%), and pain with sexual intercourse (72\%). Slightly fewer stated they were comfortable discussing lubricant use (69\%) and correct condom use (66\%). Notably, only half (50\%) stated they were comfortable discussing consent to sex for residents with dementia.

\section{Additional Comments}

Participants were asked for any additional insights regarding STDs in LTCFs. Five respondents provided comments, with 3 highlighting the need for more education in this area. One respondent commented, "This has opened my eyes to a potential need for education at this facility." Another stated, "In my opinion, if the center leaders approach this with residents and staff we may be able to decrease STDs in the entire community." An additional respondent said, "It is the residents right to engage in sexual activity if they want. Sometimes nurses and family members oppose their resident or family engaging in sexual activities-that's our highest barrier. STD education would be a good program for residents, staff, and family."

\section{Table 3. Perceived Barriers to Providing STD Education}

\begin{tabular}{|c|c|c|}
\hline Barrier & $\begin{array}{l}\text { Not a barrier or minor barrier } \\
\mathrm{N}(\%)\end{array}$ & $\begin{array}{l}\text { Moderate or major barrier } \\
\mathrm{N}(\%)\end{array}$ \\
\hline Family opposition to sex among residents & $11(34)$ & $20(63)$ \\
\hline $\begin{array}{l}\text { Lack of education among LTCF nurses regarding the prevalence of STDs in } \\
\text { older adults }\end{array}$ & $14(44)$ & $17(53)$ \\
\hline Negative nurse attitudes towards sex among residents & $16(50)$ & $14(44)$ \\
\hline Lack of training on how to address STDs among residents & $17(53)$ & $13(41)$ \\
\hline Lack of funding for STD education programming & $18(56)$ & $13(41)$ \\
\hline Nurse embarrassment regarding talking about sex with residents & $20(63)$ & $10(31)$ \\
\hline
\end{tabular}

Note: Percentages may not equal $100 \%$ due to rounding and/or nonreported answers. 
Table 4. Perceived Benefits to Providing STD Education

\begin{tabular}{|lll|}
\hline Benefit & Not a benefit or minor benefit & $\begin{array}{l}\text { Moderate or major benefit } \\
\text { N (\%) }\end{array}$ \\
\hline Being a leading LTCF for healthy initiatives & $8(25)$ & $21(66)$ \\
Promotion of healthy sexual relationships among residents & $11(34)$ & $18(56)$ \\
Improved overall health among residents & $14(44)$ & $15(47)$ \\
Improved knowledge of STDs among residents & $14(44)$ & $15(47)$ \\
Decreased STD rates in my facility & $27(85)$ & $1(3)$ \\
Other & $1(3)$ & $0(0)$ \\
\hline
\end{tabular}

Note: Percentages may not equal $100 \%$ due to rounding and/or nonreported answers.

Two additional nurses commented on the need for consent among residents by remarking the following: "Must be consensual and residents must have a high BIMS [brief interview for mental status] score" and "this topic is under spoken about in LTC especially considering the dementia population is unable to knowingly give consent to participate in sexual activity. Married couples and those able to safely consent are spoken to by social workers to ensure safety and privacy."

\section{DISCUSSION}

Nurses in our study were nearly unanimous in disagreeing STDs and HIV are problems among their residents. While there was stronger agreement that STDs and HIV are problems among older adults in the general population, this still represents a disconnect between what the nurses are perceiving as health problems in their residents and what the literature shows are growing health concerns among older adults.1-3 This corroborates previous research findings which showed that while LTCF staff consider privacy, consent, and appropriateness of sexual intimacy among residents, STDs may not be a significant concern. ${ }^{9}$ Since nurses are typically the ones assessing and triaging patients in a nursing home, perceptions could be driven by personal experience such that nurses are not observing STDs in their residents and thus do not consider such diseases a problem. Given that our study did not ask about STD screening procedures, it is unknown how nurses in nursing homes would be made aware of an STD other than patient signs and symptoms. Many STDs such as chlamydia and gonorrhea are asymptomatic, however, and thus would not be obvious without systematic screening.

Our findings also show that STD education for nursing home residents remains a rare occurrence. Just 2 respondents (6\%) stated their facility provides some sort of STD education. This should not be surprising given that previous research has demonstrated a reluctance of health care providers to discuss sexual health in older adults coupled with complex or nonexistent intimate partner policies in LTCFs. ${ }^{9-19}$ Respondents in our study also stated perceived family opposition would be the most significant barrier to providing STD education, which means decisions of care are not happening based on patient need alone. Decisions to educate residents on STDs and healthy sexual relationships are clearly a complex decision that combines nurse comfortableness, previous training, facility policies, perceptions of resident embarrassment, and perceptions of family opposition.

Surprisingly, the most significant perceived benefit to providing STD education to residents noted by respondents was the ability to be seen as a leading LTCF for healthy initiatives. Respondents in our study did not perceive a reduction in STDs to be a significant benefit, most likely due to the perception that STDs are not a problem among residents. These responses could be driven by the supervisory role of our respondents, since directors of nursing typically have responsibilities that go beyond direct patient care. ${ }^{12}$ Highlighting this benefit to nurse administrators may encourage more facilities to adopt an STD education program.

In terms of consent to sexual intimacy, dementia is often overlooked as an increasingly common diagnosis that impacts sexual expression and consent.26-29 Only half of respondents in our study said they were comfortable talking about consent to sex for residents with dementia. In any case involving sexual expression and intimacy, the individual's autonomy, privacy, and safety are the most important considerations for LTCFs. ${ }^{28}$ A person's ability to make decisions regarding their sexuality typically comes from being deemed an autonomous individual by a facility staff member conducting an assessment evaluating their cognitive abilities, known as the mini mental status exam (MMSE). ${ }^{26,28}$ Research suggests that in order for an individual with dementia to have sex without the capacity for consent, the resident, staff, and family should be involved in making decisions to keep the resident's best interest in mind. ${ }^{27}$ While these patients may have diminished thinking capabilities, it does not mean that every scenario is the same and should follow the same guidelines. ${ }^{26}$ As previous research has noted, directors of nursing in LTCFs prefer a top-down approach to addressing sexual expression, with more direction on consent and intimacy policies coming from national organizations such as CMS. ${ }^{12}$ 
Furthermore, at least 1 respondent in our study made a comment regarding how practices may differ between assisted living facilities and nursing homes since independence differs greatly based on the type of facility. Individuals living in resident communities, where they are essentially autonomous residents, have less mobility limitations and more independence in terms of day-to-day activities. As previously noted, program and service offerings in assisted living facility services can vary greatly and are not welldefined in Ohio. Since our study did not seek out responses from nurses working in assisted living facilities, findings do not represent the full spectrum of nurse perceptions in LTCFs. Future research should compare differences in nurse perception based on the type of facility.

Our study was limited by several factors. First, since this was a pilot study, the small sample size does not allow for generalizability to a broader population. Homogeneity in responses to our survey items also limited variation and comparison among study variables. A larger sample inclusive of all LTCFs, including assisted living facilities, would aid in comparison of facility demographic variables, nurse demographic variables, and perceptions of STD education. Survey items were also monothematic in nature and somewhat controversial which can influence response bias.

\section{PUBLIC HEALTH IMPLICATIONS}

Among nurses in our study, there was clear support for STD education and facilitation of healthy sexual relationships, yet implementation of STD education remains rare. Nurses report lacking the training necessary to talk with residents about STD prevalence and prevention but feel overwhelmingly comfortable talking about many sexual health concerns. While perceptions of barriers and benefits do not always translate into practice, this study expands our understanding of the hesitancy among medical professionals to discuss STD risk with residents in LTCFs.

\section{REFERENCES}

1. American Association of Retired People. National poll on healthy aging. Accessed November 15, 2019. https://www.healthyagingpoll.org

2. Syme ML, Cohn J, Barnack-Tavalaris J. A comparison of actual and perceived sexual risk among older adults. J Sex Res. 2017;54(2):149-160. https://doi.org/10.1080/00224499.2015.1124379

3. Athena health. Published May 16, 2018. Accessed November 11, 2019. https://www.athenahealth.com/insight/over-60-stis-may-not-bedone-you

4. Villar F, Celdran M, Faba J, Serrat R. Barriers to sexual expression in residential aged care facilities (RACFs): comparison of staff and residents' views. JAdv Nurs. 2014;70(11):2518-2527. https://doi.org/10.1111/jan.12398

5. Bauer M, Fetherstonhaugh D, Tarzia L, Nay R, Wellman D, Beattie E. "I always look under the bed for a man." Needs and barriers to the expression of sexuality in residential aged care: The views of residents with and without dementia. Psychol Sex. 2013;4(3):296-309. https://doi.org/10.1080/19419899.2012.713869
6. Mathieu L, Gastmans C. Older residents' perspectives on aged sexuality in institutionalized elderly care: A systematic review. Int J Nurs Stud. 2015;52:1891-1905.

https://doi.org/10.1016/j.ijnurstu.2015.07.007

7. Centers for Disease Control and Prevention (2017). Nursing home care. Accessed October 30, 2019.

https://www.cdc.gov/nchs/fastats/nursing-home-care.htm

8. Lindau ST, Schumm LP, Laumann EO, Levinson W, O'Muirchesartaigh CA, Waite LJ. A study of sexuality and health among older adults in the United States. N Engl J Med. 2007,357:762-774. https://doi.org/10.1056/NEJMoa067423

9. Gilmer MJ, Meyer A, Davidson J, Koziol-McLain J. Staff beliefs about sexuality in aged residential care. Nurs Prax N Z. 2010;26(3):17-24.

10. Penwell-Waines L, Wilson CK, Macapagal KR, et al. Student perspectives on sexual health: implications for interprofessional education. $J$ Interprof Care. 2014;28(4):317-322. https://doi.org/10.3109/13561820.2014.884553

11. Benoot C, Enzlin P, Peremans L, Bilsen J. Addressing sexual issues in palliative care: A qualitative study on nurses' attitudes, roles and experiences. JAdv Nurs. 2018;74(7):1583-1594. https://doi.org/10.1111/jan.13572

12. Syme ML, Lichtenberg P, Moye J. Recommendations for sexual expression management in long-term care: a qualitative needs assessment. $J$ Adv Nurs. 2016;72(10):2457-2467. https://doi.org/10.1111/jan.13005

13. Slinkard MS, Kazer MW. Older adults and HIV and STI screening: the patient perspective. Geriatr Nurs. 2011;5(32):341-349. https://doi.org/10.1016/j.gerinurse.2011.05.002

14. Syme ML, Yelland E, Cornelison L, Poey JL, Krajicek R, Doll G. Content analysis of public opinion on sexual expression and dementia: implications for nursing home policy development. Health Expect. 2016;20 (4):705-713. https://doi.org/10.1111/hex.12509

15. Ports KA, Barnack-Tavalaris JL, Syme ML, Perera RA, Lafata JE. Sexual health discussions with older adult patients during periodic health exams. J Sex Med. 2014;11:901-908. https://doi.org/10.1111/jsm.12448

16. Hughes AK. HIV knowledge and attitudes among providers in aging: results from a national survey. AIDS Patient Care STDS. 2011;25 (9):539-545.

https://doi.org/10.1089/apc.2011.0026

17. Villar F, Celdran M, Faba J, Serrat R. Staff members' perceived training needs regarding sexuality in residential aged care facilities. Gerontol Geriatr Educ. 2017;38(4):443-452. https://doi.org/10.1080/02701960.2015.1127811

18. Di Napoli EA, Breland GL, Allen RS. Staff knowledge and perceptions of sexuality and dementia of older adults in nursing homes. J Aging Health. 2013;25(7): 1087-1105. https://doi.org/10.1177/0898264313494802

19. American Medical Directors Association. Elder care sex survey. Published October 28, 2013. Accessed August 10, 2020. https://www.bloomberg.com/graphics/infographics/elder-care-sexsurvey-results.html

20. Bauer M, McAuliffe L, Nay R, Chenco C. Sexuality in older adults: effect of an education intervention on attitudes and beliefs of residential 
aged care staff. Educ Gerontol. 2013;39(2):82-91.

https://doi.org/10.1080/03601277.2012.682953

21. Rosenstock IM. The health belief model and preventive health behavior. Health Educ Monogr. 1974;2(4):354-386.

22. Hochbaum GM. Public Participation in Medical Screen Programs: A Socio-psychological Study. US Department of Health, Education, and Welfare: Washington, DC; 1958.

23. Maziarz LN, Dake JA, Glassman T, Telljohann SK, Beekley C. Superintendents perceptions of condom availability in US high schools. Health Behav Policy Rev. 2017;4(4):389-398.

24. Reindl D, Glassman T, Price J, Dake J, Yingling F. Perceptions of college and university presidents regarding tobacco-free campus policies. Jof Am Coll Health, 2014.62(3):193-202.

https://doi.org/10.1080/07448481.2013.877019

25. Centers for Medicare and Medicaid Services (CMS) Skilled Nursing Facilities Report. Published February 2, 2021. Accessed March 30, 2021.

https://data.cms.gov/provider-data/dataset/4pq5-n9py

26. Victor E, Guidry-Grimes L. Relational autonomy in action: rethinking dementia and sexuality in care facilities. Nurs Ethics. 2019;26(6):16541664.

https://doi.org/10.1177/0969733018780527

27. Wilkins JM. More than capacity: alternatives for sexual decision making for individuals with dementia. Gerontologist. 2015;55(5):716-723. https://doi.org/10.1093/geront/gnv098

28. Hillman J. Sexual consent capacity: ethical issues and challenges in long-term care. Clin Gerontol. 2016;40(1):43-50.

https://doi.org/10.1080/07317115.2016.1185488

29. Cornelison LJ, Doll GM. Management of sexual expression in long-term care: ombudsmen's perspectives. Gerontologist. 2012;53(5):

780-789.

https://doi.org/10.1093/geront/gns162 